\title{
Nontuberculous mycobacterial infection after lung transplantation: a report of four cases
}

\author{
Naoko Ose ${ }^{*} \mathbb{D}$, Masato Minami, Soichiro Funaki, Takashi Kanou, Ryu Kanzaki and Yasushi Shintani
}

\begin{abstract}
Background: Nontuberculous mycobacterium (NTM) infection in a patient in an immunosuppressed state caused by increased use of immunosuppressive or biological agents is a serious clinical problem. Mycobacterium avium complex is the most common involved pathogen, followed by Mycobacterium abscessus (MABSC), while Mycobacterium kansasii is not a major concern. The rate of infection rate in lung transplant recipients is reported to range from $1.5-22.4 \%$.

Case presentation: We report here four cases of NTM pulmonary infection and disease among 63 patients who underwent lung or heart-lung transplantation at our hospital. Those four occurred following living-donor transplantation in two patients, one with pulmonary arterial hypertension and one with bronchiectasia, and deceased donor lung transplantation in two patients, lymphangioleiomyomatosis and interstitial pneumonia, respectively. NTM was not detected in any of the patients prior to transplantation. The involved pathogens were Mycobacterium gordonae in one, MAC in one, and MABSC in two of these patients, which were isolated from broncho-alveolar lavage (BAL) in two and sputum in two. The one case of MAC and two of MABSC were symptomatic with consolidation shown in chest $C T$ images indicating possible pneumonia, while the one with M. gordonae had no symptoms and was detected by surveillance BAL. Onset time from detection of NTM was greater than 3 years in the three with MABSC and M. gordonae and less than 3 years in the one with MAC. Each patient required a decrease in immunosuppressive agents according to their condition, while antibiotics therapy was performed in the three who were symptomatic. Sputum culture findings became negative after several months and were maintained thereafter in all.

Conclusion: An NTM infection leading to pulmonary disease can occur at any time following lung transplantation. Treatment should be considered depending on the involved pathogens, individual status, and disease severity.
\end{abstract}

Keywords: Nontuberculous mycobacteriosis, NTM, Infection, Lung, Transplantation

\section{Background}

The number of patients with pulmonary nontuberculous mycobacterium (NTM) is increasing worldwide [1, 2], with NTM infection in patients in an immunosuppressed state caused by increased use of immunosuppressive or biological agents a serious clinical problem. Isolation of NTM organisms following lung transplantation is reported to occur in 1.5-22.4\% [3-11] of those patients, some of whom suffer from pulmonary disease. The most

\footnotetext{
* Correspondence: naokoose@thoracic.med.osaka-u.ac.jp Department of General Thoracic Surgery, Osaka University Graduate School of Medicine, 2-2 Yamadaoka, Suita-shi, Osaka, Japan
}

common strain varies among regions and countries, which is an important factor because effective treatment methods differ depending on the causative pathogen. Furthermore, onset period and infection site in the native or transplanted lung are critical for effective treatment.

We report here four cases of NTM pulmonary infection in patients who underwent lung transplantation and were treated at our institution and also review previous reports with focus on onset time and NTM strain. Of 63 patients at our hospital who underwent lung transplantation between January 2000 and January 2018, including heart-lung and living-donor transplantation, four $(6.3 \%)$ had NTM 
isolated from obtained specimens, while NTM was not detected in any prior to transplantation. Surveillance bronchoscopy examinations were performed four times the first year and then annually until 5 years after transplantation, according to our institutional protocol. After the fifth year, clinically indicated bronchoscopic examination is occasionally performed when rejection or respiratory infection suspects. In the present cases, clinical samples were obtained from sputum and bronchial lavage samples obtained with bronchoscopy. Mycobacterial cultures were performed using Ogawa medium or a Mycobacteria Growth Indicator Tube system. No Mycobacterium (M.) tuberculosis organisms were isolated in any of the present cases.

\section{Case presentations}

\section{Case 1}

An 11-year-old boy with pulmonary arterial hypertension underwent living-donor lung transplantation, with tacrolimus, mycophenolate mofetil (MMF), and prednisolone (PSL) given as immunosuppressive agents. At 76 months after transplantation, M. gordonae was isolated from a broncho-alveolar lavage (BAL) sample obtained during a surveillance examination. Contamination was suspected, because even though there were no symptoms, chest computed tomography (CT) showed a slight amount of consolidation in the left upper lesion (Fig. 1a). PSL as therapeutic and diagnostic treatment was decreased. Sputum culture findings were negative after 5 months, and chest CT images were clear. There was no further NTM detection during the following 15 years.

\section{Case 2}

A 38-year-old female with bronchial ectasia underwent living-donor lung transplantation, with ciclosporin, MMF, and PSL given as immunosuppressive agents. At 82 months after transplantation, the patient developed a fever with purulent sputum and chest CT showed consolidation in the left lower lesion (Fig. 1b). M. abscessus complex (MABSC) was isolated from a BAL sample. Following administrations of tazobactam/piperacillin and azithromycin (AZM), as well as a decrease in ciclosporin from 120 to $50 \mathrm{mg}$ for 1 month, the sputum cultures became negative. AZM administration and decreased ciclosporin were continued for 22 months, with no recurrence noted.

\section{Case 3}

A 39-year-old female with lymphangioleiomyomatosis underwent single deceased donor lung transplantation, with ciclosporin, MMF, and PSL given as immunosuppressive agents. At 58 months after transplantation, a fever developed and chest CT showed consolidation in the transplanted lung (Fig. 1c). MABSC was isolated in a cultured sputum sample. Following administrations of imipenem, amikacin, and AZM for 4 months, sputum culture findings became negative. Maintenance therapy with imipenem and amikacin was given once a week, along with daily AZM and a decrease in MMF, with no recurrence seen during the following 1-year period.

\section{Case 4}

A 41-year-old male with interstitial pneumonia underwent single deceased donor lung transplantation, with

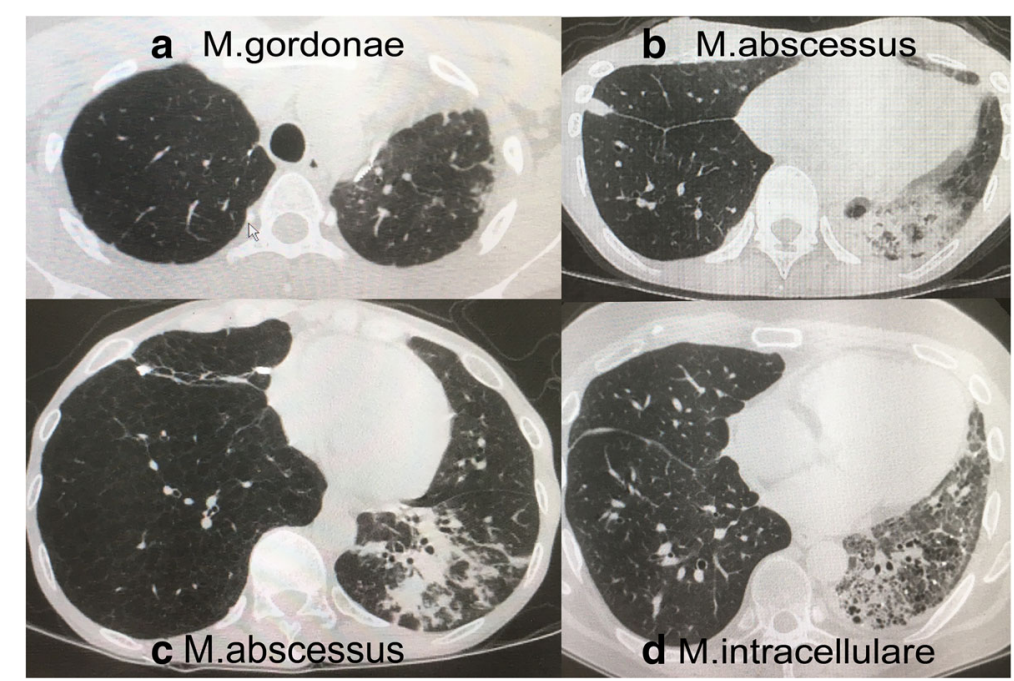

Fig. 1 a-d. Chest computed tomography showing consolidation. a Case 1, slight consolidation in left upper lesion caused by M. gordonae. $\mathbf{b}$ Case 2, consolidation in left lower lesion caused by M. abscessus. c Case 3, consolidation in transplanted lung caused by M. abscessus. d Case 4, consolidation in native lung caused by $M$. intracellulare 


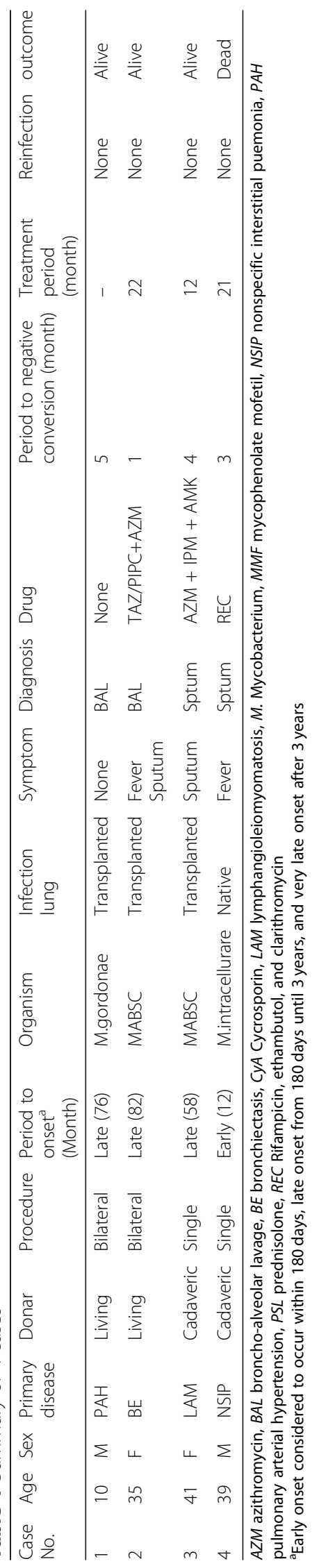


Table 2 Previous reports of NTM lung infection after lung transplant

\begin{tabular}{|c|c|c|c|c|c|c|}
\hline \multirow{2}{*}{\multicolumn{2}{|c|}{ references }} & \multirow{3}{*}{$\begin{array}{l}\text { NTM lung infection } \\
6.5 \%(17 / 261)\end{array}$} & \multirow{3}{*}{\begin{tabular}{|l} 
MAC/MABSC/Others** \\
$(\%)$
\end{tabular}} & \multicolumn{3}{|c|}{ Onset time ${ }^{\dagger}$} \\
\hline & & & & \multirow{2}{*}{$\frac{\text { early }}{3}$} & \multirow{2}{*}{$\frac{\text { late }}{8}$} & \multirow{2}{*}{$\frac{\text { very late }}{6}$} \\
\hline 1999 & Malouf $^{3)}$ & & & & & \\
\hline 1999 & Kesten $^{4)}$ & $2.7 \%(6 / 219)$ & $50 / 0 / 50$ & 8 & 0 & 0 \\
\hline 2002 & Fairhurst $^{5)}$ & $1.5 \%(3 / 261)$ & $0 / 3 / 0$ & 1 & 1 & 1 \\
\hline 2006 & Chernenko $^{6}$ & $0.28 \%(15 / 5200)^{*}$ & only MABSC & 5 & 5 & 5 \\
\hline 2011 & Huang $^{7)}$ & $18.0 \%(36 / 201)$ & $61 / 14 / 25$ & 22 & 14 & 0 \\
\hline 2012 & Knoll $^{8)}$ & $22.4 \%(53 / 237)$ & 70/9.4/20.6 & $3^{\S}$ & $2^{\S}$ & $1^{\S}$ \\
\hline 2016 & Shah $^{9)}$ & $14.4 \%(30 / 208)$ & $36 / 46 / 18$ & - & - & - \\
\hline 2016 & George $^{10)}$ & $7.7 \%(43 / 553)$ & $58 / 6 / 36$ & - & - & - \\
\hline \multirow[t]{2}{*}{2018} & Tachibana $^{11)}$ & $2.1 \%(5 / 240)$ & $33 / 20 / 47$ & 1 & 4 & 0 \\
\hline & Our cases & $6.6 \%(4 / 61)$ & $25 / 50 / 25$ & 0 & 1 & 3 \\
\hline \multicolumn{7}{|c|}{$\begin{array}{l}\text { * infection rate of only M ABSC not NTM } \\
\text { **Others included M.kansasii, M.gordonae, M.xenopi, M.chelonae, M.siminae, M.fortuitum, M.peregrinum, } \\
\text { M.asiaticum, M.terrae, M.mucogenicum, M.immunogenum, M.smegmatis and M.lentiflavum. } \\
\dagger \text { early onset considered to occur within } 180 \text { days, late onset from } 180 \text { days until } 3 \text { years, and very late onset after } 3 \text { years. } \\
\S \text { No mentions about onset time in anothor cases. }\end{array}$} \\
\hline
\end{tabular}

ciclosporin, MMF, and PSL given as immunosuppressive agents. At 12 months after transplantation, a fever developed and chest CT showed consolidation in the native lung (Fig. 1d), which was suspected to be pneumonia caused by general bacteria. Broad-spectrum antibiotic therapy was started, though was not effective. After a period of time, $M$. intracellulare was isolated from a cultured sputum sample. Thereafter, rifampicin (RFP), ethambutol, and clarithromycin (REC) treatment was administered for 3

Table 3 Pathogens classified by onset time

\begin{tabular}{llll}
\hline & $\begin{array}{l}\text { Early } \\
(n=43)\end{array}$ & $\begin{array}{l}\text { Late } \\
(n=35)\end{array}$ & $\begin{array}{l}\text { Very late } \\
(n=16)\end{array}$ \\
\hline MAC $^{\text {a }}$ & 4 & 13 & 5 \\
MABSC $^{b}$ & 11 & 10 & 9 \\
M.gordonae & 1 & 0 & 1 \\
M.chelonae & 1 & 0 & 0 \\
M.siminae & 0 & 1 & 0 \\
M.kansasii & 0 & 1 & 0 \\
M.fortuitum & 0 & 3 & 0 \\
M.asiaticum & 0 & 0 & 1 \\
Not reported & 26 & 7 & 0 \\
\hline
\end{tabular}

${ }^{\mathrm{a}} \mathrm{MAC}$ mycobacterium avium complex

${ }^{\mathrm{b}}$ MABSC Mycobacterium abscessus complex months, after which sputum findings were negative. Rifampicin was continued at $800 \mathrm{mg}$, the standard level, while ciclosporin was adjusted according to trough level and finally administered at $550-600 \mathrm{mg}$, three times the normal dose, and PSL was gradually reduced. The patient died after 21 months because of respiratory failure due to chronic rejection, though sputum culture findings remained negative with REC treatment These cases are summarized in Table 1.

\section{Discussion}

The number of patients worldwide with pulmonary NTM disease is increasing [1, 2]. An NTM infection is not contagious, whereas an MABSC infection can be acquired through transmission, potentially via fomites or aerosols [12]. Floto et al. reported that an NTM infection occurring in patients with cystic fibrosis can be problematic, as that can influence the indication for lung transplantation [13].

NTM infection following transplantation is a critical issue, because treatment is difficult, especially for patients in an immunosuppressed condition, as there is no specific medicine available for NTM, unlike $M$. tuberculosis. In hematopoietic stem cell or other solid organ recipients, the most common site of infection is the lung, with M. avium complex (MAC), the most 


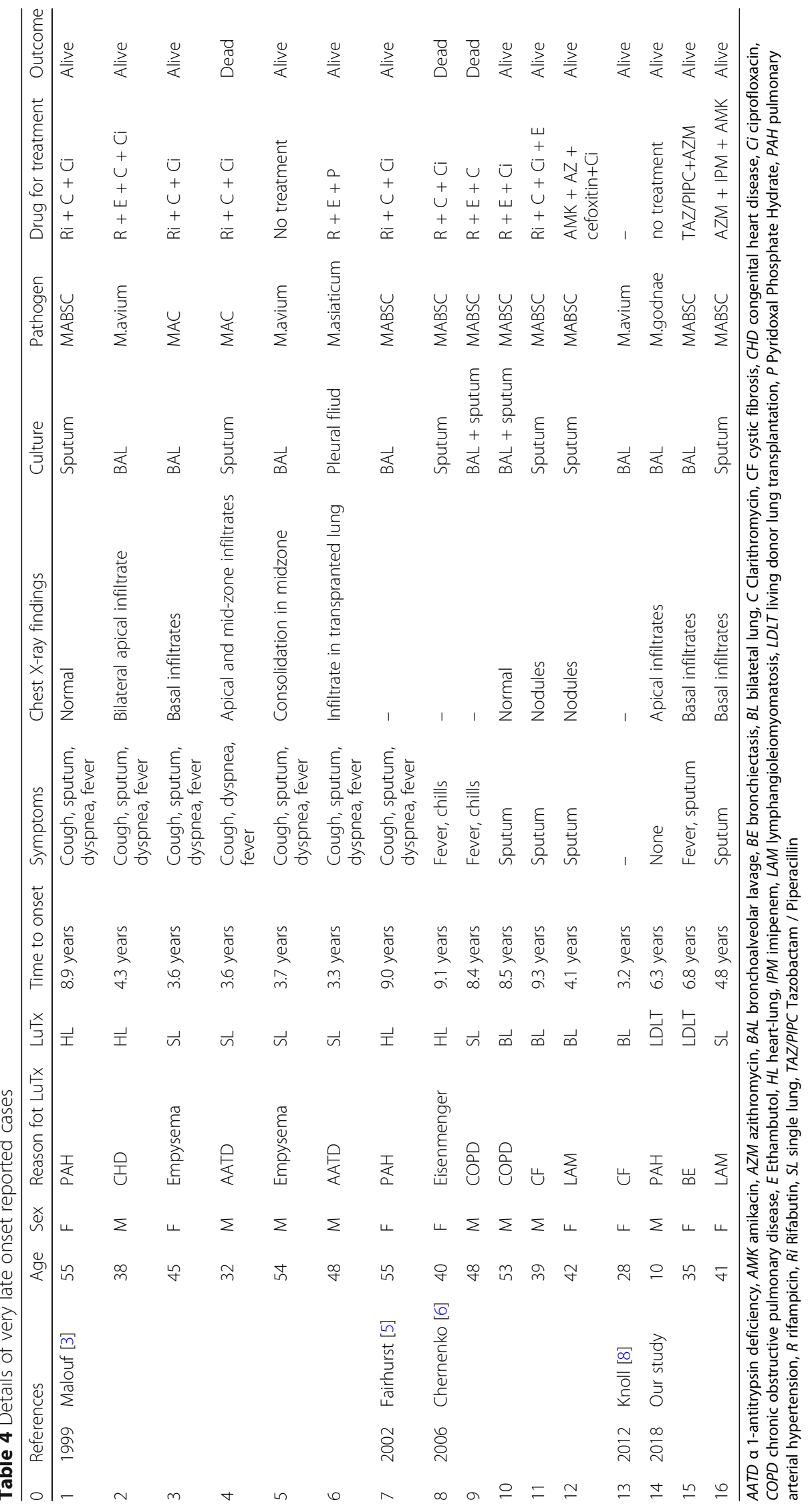


commonly noted causative pathogen, followed by $M$. kansasii [14, 15]. Other studies have suggested that NTM infection rates of incidence in lung transplant recipients range from $1.5-22.4 \%$ (Table 2) [3-11]. In those reports, MAC was most common, followed by MABSC, while M. kansasii was not a major concern. However, there are also reports of rare types of NTM (Table 3), though the causative pathogens vary depending on region. In those cases, the most common infection site is a lung, followed by skin and soft tissue caused by rapid growers, such as MABSC or $M$. gordonae.

We reviewed previous reports and classified those cases based on onset time after transplantation, with early onset considered to occur within 180 days, late onset from 180 days until 3 years, and very late onset after 3 years. There were 212 cases in which onset time was described (early onset, $n=43$; late onset, $n$ $=35$; very late onset, $n=16$ ) (Table 2), including 61 with both types of pathogens and onset time details (Table 3). MAC and MABSC were common organisms regardless of time of onset. In cases of early onset, the origin of NTM was newly acquired in some and prior to transplantation or donor-derived in others. On the other hand, patients in the very late onset group acquired a new infection under an immunosuppressed condition. All 16 cases had respiratory symptoms due to NTM pulmonary disease (Table 4). The average time from transplantation to onset was 6.1 years, and prognosis in most was good (Table 4). The direct cause of the three mortalities was not NTM, but rather bronchiolitis obliterans syndrome (BOS) in one [3], and either aspergillus or pneumonia infection in two [6].

Chronic rejection is known to be associated with NTM infection [3], and bronchiolitis obliterans syndrome is likely to occur in patients with NTM detected [7, 9]. Most of the patients in our cohort had respiratory symptoms, such as cough, sputum, and fever, with imaging showing consolidation suggesting pneumonia, and were diagnosed based on sputum culture or BAL results. Aggravation of a pulmonary infection in cases affected by immunosuppression will occur if an inappropriate antibiotic medicine is administered. Sputum obtained by expectoration may not be representative of sputum from deeper parts; thus, it is useful to first perform BAL to detect pathogens with the possibility of a combined infection of acid-fast bacteria or fungus kept in mind. The percentage of patients with MABSC was high (56.3\%), which included therapy resistant cases ending in death from respiratory failure, though that rate may be falsely high because of selection bias. It is important for physicians to be aware that the risk of NTM infection continues for a long period after transplantation, as that was previously reported to occur in a patient after 9 years [6], and such occurrence may be via the same mechanism seen in the general population. NTM can destroy the lung structure, leading to decreased respiratory function [12]. Thus, care must be taken regarding reduction of immunosuppressive agents because respiratory function can worsen if rejection occurs. In most reports, overall survival after lung transplantation was not influenced by NTM infection $[8,9]$, though George et al. found that the survival rate of pulmonary NTM disease was lower than that of subclinical infection cases [10]. On the other hand, another investigation found that it was similar between cases of single lung transplantation and those with BOS [7].

Selection of the optimum antibiotic medication for treatment is crucial, though dependent on the sensitivity of each pathogen according to the ATS guidelines and must also be adjusted in accordance with the prescribed immunosuppressive agents. On the other hand, that is dependent on individual patient state regarding which agents can be reduced. PSL was decreased in two of our cases and only case 1 showed improvement with treatment. RFP, administered for MAC, attenuates the effect of ciclosporin; thus, it is necessary to increase its dose by 3-5 times above the standard when administered. The present case 4 was given an approximately threefold greater dose of ciclosporin while receiving the standard RFP dosage. Ciclosporin must be adjusted by monitoring its concentration in blood, as that varies in each patient from a variety of factors.

\section{Conclusions}

An NTM infection and pulmonary disease can occur several years following lung transplantation. It is necessary to consider the best treatment for affected patients based on the pathogens involved, individual status, and disease severity, including which antibiotic medicine should be selected and which immunosuppressive agent can be decreased.

\footnotetext{
Abbreviations

AATD: a-1 antitrypsin deficiency; AMK: Amikacin; AZM: Azithromycin; BAL: Broncho-alveolar lavage; BE: Bronchiectasis; BL: Bilatetal lung; BOS: Bronchiolitis obliterans syndrome; C: Clarithromycin; CF: Cystic fibrosis; CHD: Congenital heart disease; Ci: Ciprofloxacin; COPD: Chronic obstructive pulmonary disease; CT: Computed tomography; CyA: Ciclosporin; E: Ethambutol; HL: Heart-lung; IPM: Imipenem;

LAM: Lymphangioleiomyomatosis; LDLT: Living donor lung transplantation; M.: Mycobacterium; MABSC: Mycobacterium abscessus; MAC: Mycobacterium avium complex; MMF: Mycophenolate mofetil; NSIP: Nonspecific interstitial pneumonia; NTM: Nontuberculous mycobacteriosis; P: Pyridoxal phosphate hydrate; PAH: Pulmonary arterial hypertension; PSL: Prednisolone; R: Rifampicin; REC: Rifampicin, ethambutol, and clarithromycin; RFP: Rifampicin; Ri: Rifabutin; SL: Single lung; TAZ/PIPC: Tazobactam/ piperacillin
} 
Acknowledgements

Not applicable.

\section{Funding}

This study was not funded.

Availability of data and materials

There were no available repositories.

\section{Authors' contributions}

NO described and designed the article. YS supervised the edition of the manuscript. Other remaining co-authors collected the data and discussed the content of the manuscript. All authors read and approved the final manuscript.

\section{Ethics approval and consent to participate}

The study protocol was approved by the Ethical Review Board for Clinical Studies at Osaka University (control number 10026-3).

\section{Consent for publication}

Informed consent was obtained from the patient for the publication of this case report.

\section{Competing interests}

The authors declare that they have no competing interests.

\section{Publisher's Note}

Springer Nature remains neutral with regard to jurisdictional claims in published maps and institutional affiliations.

Received: 26 November 2018 Accepted: 3 January 2019

Published online: 23 January 2019

\section{References}

1. Adjemian J, Olivier KN, Seitz AE, Holland SM, Prevots DR. Prevalence of nontuberculous mycobacterial lung disease in U.S. Medicare beneficiaries. Am J Respir Crit Care Med. 2012;185:881-6.

2. Thomson RM. Changing epidemiology of pulmonary nontuberculous mycobacteria infections. Emerg Infect Dis. 2010;16:1576-83.

3. Malouf MA, Glanville AR. The spectrum of mycobacterial infection after lung transplantation. Am J Respir Crit Care Med. 1999;160:1611-6.

4. Kesten S, Chaparro C. Mycobacterial infections in lung transplant recipients. Chest. 1999;115:741-5.

5. Fairhurst RM, Kubak BM, Shpiner RB, Levine MS, Pegues DA, Ardehali A. Mycobacterium abscessus empyema in a lung transplant recipient. J Heart Lung Transplant. 2002:21:391-4.

6. Chernenko SM, Humar A, Hutcheon M, Chow CW, Chaparro C, Keshavjee S, et al. Mycobacterium abscessus infections in lung transplant recipients: the international experience. J Heart Lung Transplant. 2006;25:1447-55.

7. Huang HC, Weigt SS, Derhovanessian A, Palchevskiy V, Ardehali A, Saggar R, et al. Non-tuberculous mycobacterium infection after lung transplantation is associated with increased mortality. J Heart Lung Transplant. 2011;30:790-8.

8. Knoll BM, Kappagoda S, Gill RR, Goldberg HJ, Boyle K, Baden LR, et al. Nontuberculous mycobacterial infection among lung transplant recipients: a 15year cohort study. Transpl Infect Dis. 2012;14:452-60.

9. Shah SK, McAnally KJ, Seoane L, Lombard GA, LaPlace SG, Lick S, et al. Analysis of pulmonary non-tuberculous mycobacterial infections after lung transplantation. Transpl Infect Dis. 2016;18:585-91.

10. George IA, Santos CA, Olsen MA, Bailey TC. Epidemiology and outcomes of nontuberculous mycobacterial infections in solid organ transplant recipients at a Midwestern center. Transplantation. 2016;100:1073-8.

11. Tachibana K, Okada Y, Matsuda Y, Miyoshi K, Oto T, Chen-Yoshikawa TF, et al. Nontuberculous mycobacterial and Aspergillus infections among cadaveric lung transplant recipients in Japan. Respir Investig. 2018;56:243-8.

12. Bryant JM, Grogono DM, Rodriguez-Rincon D, Everall I, Brown KP, Moreno P, et al. Emergence and spread of a human-transmissible multidrug-resistant nontuberculous mycobacterium. Science. 2016;354:751-7.

13. Floto RA, Olivier KN, Saiman L, Daley CL, Herrmann JL, Nick JA, et al. US Cystic Fibrosis Foundation and European Cystic Fibrosis Society consensus recommendations for the management of nontuberculous mycobacteria in individuals with cystic fibrosis. Thorax. 2016;71:88-90.
14. Patel R, Roberts GD, Keating MR, Paya CV. Infections due to nontuberculous mycobacteria in kidney, heart, and liver transplant recipients. Clin Infect Dis. 1994;19:263-73.

15. Queipo JA, Broseta E, Santos M, Sánchez-Plumed J, Budía A, Jiménez-Cruz F. Mycobacterial infection in a series of 1261 renal transplant recipients. Clin Microbiol Infect. 2003;9:518-25.

\section{Submit your manuscript to a SpringerOpen ${ }^{\circ}$ journal and benefit from:}

- Convenient online submission

- Rigorous peer review

- Open access: articles freely available online

- High visibility within the field

- Retaining the copyright to your article

Submit your next manuscript at $\boldsymbol{\nabla}$ springeropen.com 